\title{
DRENAGEM ÁCIDA EM MATERIAIS PROVENIENTES DA MINERAÇÃO DE CARVÃO, DE CANDIOTA RS
}

\author{
Evaldo R. Soares(*), Jaime W. V. de Mello(**), \\ Liovando M. da Costa(**) \& Mauro L. T. Corrêa(***)
}

\begin{abstract}
An experiment was carried out to study the heavy metals mobility and the use of clay layers with or without $\mathrm{CaCO}_{3}$ as treatments for reducing drainage water acidification and heavy metal contamination of materials from coal mining. The materials utilized were mine wastes, coal ashes and mudstones from a mining region in Candiota-RS. These materials were placed into leaching columns, and subjected to percolation. After leaching, $\mathrm{pH}, \mathrm{Ni}, \mathrm{Pb}, \mathrm{Mn}, \mathrm{Zn}$ and $\mathrm{Fe}$ contents were determined in the leached solution. It was observed that: (a) there is heavy metals mobilization with potential to contaminate the drainage water, (b) ashes are not the unique source of contamination, (c) clay layers were not efficient to reduce contamination. Otherwise, the use of $\mathrm{CaCO}_{3}$ was efficient to prevent the metal mobilization in drained water.

Key-words: Coal; Heavy metals; Acid drainage, Mobility.
\end{abstract}

\section{INTRODUÇÃO}

A mina de Candiota, localizada no RS, constitui a maior jazida de carvão mineral do Brasil, com reservas estimadas em torno de 8,5 bilhões de toneladas (Brasil 1987). No entanto, a despeito de sua grande importância para a geração de energia no país, a atividade mineradora é potencialmente poluidora, o que pode representar elevado comprometimento ambiental para a região.

Geologicamente, o carvão de Candiota encontrase na formação Rio Bonito, a qual caracteriza-se por sedimentos de granulometria variável, sendo que as camadas de carvão aparecem sempre confinadas a duas camadas de argilitos (Aboarrage \& Lopes 1986). O processo de mineração, a céu aberto, requer a retirada das camadas sobrejacentes ao carvão que são misturadas durante a extração, constituindo os rejeitos.

Após a queima para a geração de energia na usina termoelétrica, as cinzas do carvão, contendo metais pesados (Zanella 1988), retornam à cava de mineração e, sobre estas, são depositados os rejeitos da cava seguinte. Vale salientar que as cinzas são depositadas sobre uma camada de arenito e que os rejeitos geralmente contêm carvão piritoso misturado. Nessas condições pode ocorrer a acidificacão (Singer \& Stumm 1970) e a mobilização dos metais pesados nas águas de drenagem e, conseqüentemente, o comprometimento dos recursos hídricos, tanto superficiais quanto subsuperficiais, próximos à área de mineração.

Nos últimos 30 anos foram minerados aproximadamente 10 milhões de toneladas de carvão, em uma área correspondente a cerca de 360 ha. A Companhia Riograndense de Mineração (CRM), responsável pela exploração do carvão de Candiota, detém o alvará de lavra de mais de 22000 ha e, de acordo com o plano 2010 da Eletrobrás, esta exploração deverá ser incrementada para 25 milhões de toneladas ao ano. Portanto, ainda que a contaminação ambiental presente não seja considerada comprometedora, preconiza-se um incremento de 75 vezes na atividade mineradora para um futuro próximo. Tendo em vista que a região caracteriza-se como produtora de arroz e carne e que estas atividades dependem dos recursos hídricos regionais, teme-se a incorporação de metais pesados na cadeia alimentar (Flores 1990). Tal perspectiva justifica plenamente os estudos no sentido de avaliar a contaminação dos parcos recursos hídricos da região e buscar alternativas para minimizá-la.

A análise da problemática permite, entre outras, as seguintes suposições: (a) - o processo de mineração e acondicionamento dos rejeitos e cinzas de combustão do carvão conduzem à contaminação das águas de drenagem da mina, com potencial para comprometer os recursos hídricos da região; (b) existe a possibilidade de minimizar a contaminação das águas de drenagem, via tratamento adequado dos rejeitos e cinzas. Nesse sentido, o uso de camadas de argila sobre e sob as cinzas e, ou o uso de calcário, misturado aos rejeitos, podem representar alternativas viáveis para neutralizar a acidez e minimizar a mobilização de metais pesados nas águas de drenagem.

Diante de tais possibilidades, elaborou-se um trabalho de simulação com materiais provenientes da área de mineração, em colunas de percolação, visando atender aos seguintes objetivos : (a) - avaliar a acidificação das águas de percolação e a mobilização de alguns metais pesados em materiais provenientes da mineração e combustão do carvão de Candiota; e (b) - verificar a eficiência técnica do uso de calcário e, ou, camadas de argila, nos rejeitos de mineração, para minimizar a contaminação das águas de percolação. 


\section{MATERIAL E MÉTODOS}

Foi montado um experimento nas dependências do Departamento de Solos da Universidade Federal de Viçosa, visando simular, em escala, a disposição das camadas de sedimentos após a mineração, segundo os tratamentos propostos. As unidades experimentais constaram de combinações de sedimentos no interior de colunas de PVC $(10 \mathrm{~cm}$ de diâmetro por $40 \mathrm{~cm}$ de altura), sendo que os sedimentos são materiais provenientes da área de mineração.

$\mathrm{Na}$ parte superior das colunas foi colocado uma camada com $25 \mathrm{~cm}$ de altura contendo $2,6 \mathrm{~kg}$ de estéreis peneirados em malha $2 \mathrm{~mm}$. Na camada correspondente às argilas, utilizaram-se $500 \mathrm{~g}$ de argila compactada em $4 \mathrm{~cm}$ de modo a obter-se a densidade de $1,6 \mathrm{~g} / \mathrm{cm}^{3}$. Na camada correspondente às cinzas utilizou-se uma espessura de $5 \mathrm{~cm}$, com $350 \mathrm{~g}$, já que a densidade aparente obtida para as cinzas foi igual a $0,9 \mathrm{~g} / \mathrm{cm}^{3}$. Na base de todas as colunas foi colocada uma camada de areia lavada, com espessura variável, dependendo do tratamento, de modo a completar os $40 \mathrm{~cm}$ de altura. A areia utilizada foi obtida da desagregação de arenitos coletados na área de mineração. Os tratamentos $\left(\mathrm{T}_{1}\right.$ a $\mathrm{T}_{11}$ ) foram definidos de acordo com as seguintes combinações, mostradas na Figura 1.

A quantidade de $\mathrm{CaCO}_{3}$ misturada nos rejeitos foi determinada considerando-se que todo o enxofre presente no rejeito estaria na forma de sulfetos. Assim, o nível de 1,0 vez a necessidade de calcário (N.C.) corresponde a uma quantidade de $\mathrm{CaCO}_{3}$ necessária para neutralizar todo o ácido capaz de se formar pela oxidação do sulfeto. Desta forma, partindo-se de 1,08 $\%$ de $\mathrm{S}$ no rejeito, obtém-se uma quantidade de 33,75 g de $\mathrm{CaCO}_{3}$ para cada $\mathrm{kg}$ de rejeito. Considerando que a quantidade de rejeitos nas colunas foi de $2,6 \mathrm{~kg}$, as doses de $\mathrm{CaCO}_{3}$ correspondentes aos níveis de calcário foram:

$$
\begin{aligned}
& \text { 0,2 N.C. }=17,55 \mathrm{~g} \text { de } \mathrm{CaCO} 3 / \text { coluna. } \\
& 0,4 \text { N.C. }=35,10 \mathrm{~g} \text { de } \mathrm{CaCO} / \text { coluna. } \\
& \text { 0,6 N.C. }=52,65 \mathrm{~g} \text { de } \mathrm{CaCO}_{3} / \text { coluna. } \\
& 0,8 \text { N.C. }=70,20 \mathrm{~g} \text { de } \mathrm{CaCO}_{3} / \text { coluna } . \\
& 1.0 \text { N.C. }=87,75 \mathrm{~g} \text { de } \mathrm{CaCO}_{3} / \text { coluna }
\end{aligned}
$$

O teor de enxofre contido nas amostras dos rejeitos, foi determinado por ataque com água régia $(3 \mathrm{HCl}$ : 1 $\mathrm{HNO}_{3}$ ) em substituição á metodologia descrita em EMBRAPA (1979). Estas colunas foram lixiviadas periodicamente com água destilada, visando estudar a mobilização de metais pesados nas águas de drenagem.

Após 30 dias da montagem das colunas foram iniciadas as análises dos lixiviados. Por um período de 4 meses foram adicionados, lentamente no topo das colunas, quantidades de água destilada equivalentes a precipitações de aproximadamente $100 \mathrm{~mm}$ a cada 15 dias $(780 \mathrm{ml})$, perfazendo um total de aproximadamente $800 \mathrm{~mm}$ durante todo o período experimental. A água percolada foi coletada na parte inferior da coluna, determinando-se o $\mathrm{pH}$ e as concentrações de $\mathrm{Pb}, \mathrm{Ni}$, $\mathrm{Zn}, \mathrm{Mn}$ e $\mathrm{Fe}$ no lixiviado. A determinação potenciométrica do $\mathrm{pH}$ foi realizada imediatamente após a coleta do lixiviado, e o teor dos metais

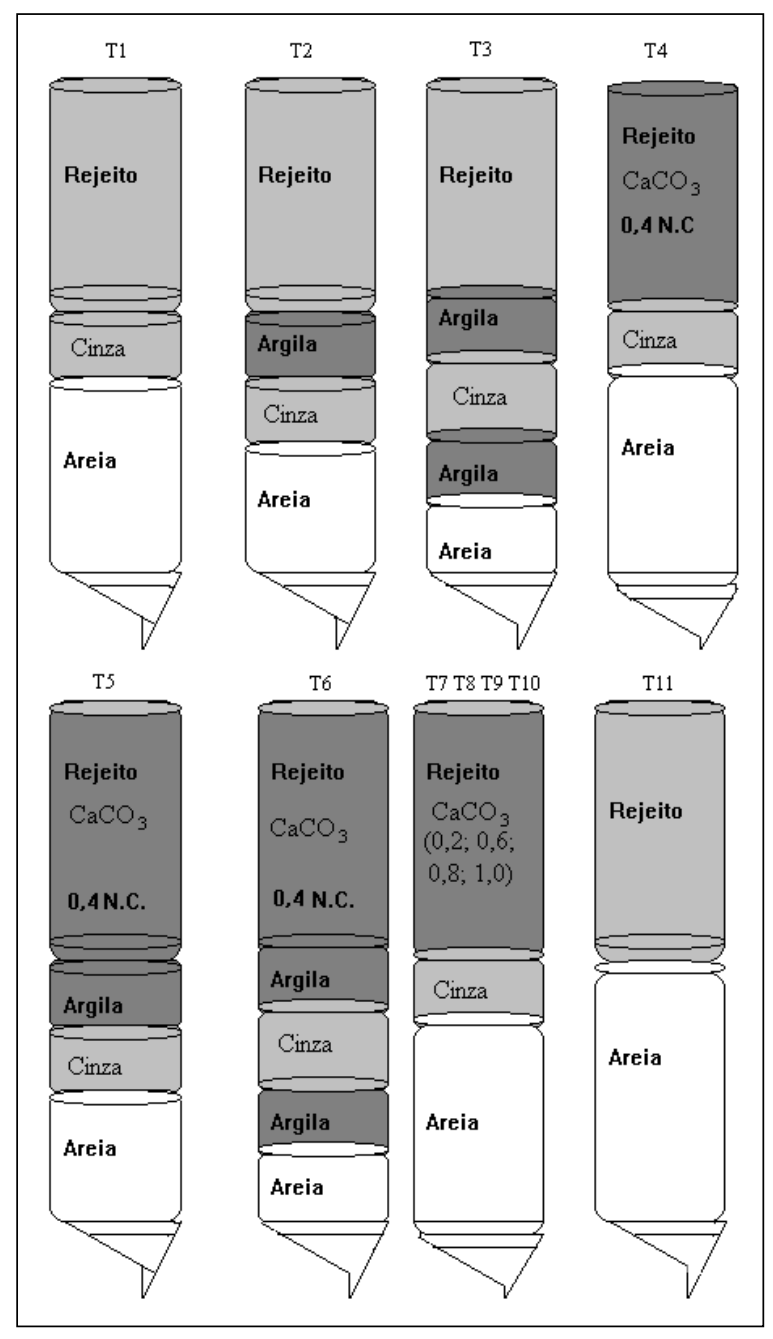

Figura 1: Representação esquemática das combinações de sedimentos nas colunas, segundo os diferentes tratamentos.

Figure 1: Schematic representation of the combined type of sediments in the colums.

analisados por espectrofotometria de absorção atômica.

O delineamento experimental adotado foi o de blocos ao acaso com três repetições. $\mathrm{Na}$ análise de variância, seis graus de liberdade para tratamentos foram desdobrados de acordo com os seguintes contrastes: $\mathrm{C}_{1}$ - Uso de camadas de argila: (tratamentos sem argila, $\mathrm{T}_{1}$ $\mathrm{T}_{4}$, versus tratamentos com argila, $\left.\mathrm{T}_{2}, \mathrm{~T}_{3}, \mathrm{~T}_{5}, \mathrm{~T}_{6}\right) ; \mathrm{C}_{2}-$ Localização das camadas de argila (tratamentos com argila sobre as cinzas , $\mathrm{T}_{2}, \mathrm{~T}_{5}$, versus tratamentos com argila sobre e sob as cinzas, $\left.\mathrm{T}_{3}, \mathrm{~T}_{6}\right) ; \mathrm{C}_{3}$ - Uso de carbonato de cálcio dentro de tratamentos sem argila (tratamento sem carbonato de cálcio e sem camadas de argila, $\mathrm{T}_{1}$, versus tratamento com carbonato de cálcio e sem camadas de argila, $\mathrm{T}_{4}$ ); $\mathrm{C}_{4}$ - Uso de carbonato de cálcio dentro de tratamentos com argila sobre as cinzas (tratamento sem carbonato de cálcio e com camada de argila sobre as cinzas, $\mathrm{T}_{2}$, versus tratamento com carbonato de cálcio e com camadas de argila sobre as cinzas, $\mathrm{T}_{5}$ ) $; \mathrm{C}_{5}$ - Uso de carbonato de cálcio dentro de tratamentos com argila sob e sobre as cinzas 
(tratamentos sem carbonato de cálcio e com camadas de argila sob e sobre as cinzas, $\mathrm{T}_{3}$, versus tratamento com carbonato de cálcio e com camadas de argila sob e sobre as cinzas, $\mathrm{T}_{6}$ ); $\mathrm{C}_{6}-$ Presença de cinzas de combustão(tratamento sem cinza, $\mathrm{T}_{11}$, versus tratamento com cinza , $\mathrm{T}_{1}$ )

Os demais graus de liberdade foram destinados ao estudo do efeito provocado pelas doses crescentes de carbonato de cálcio adicionado ao rejeito $\left(\mathrm{T}_{1}, \mathrm{~T}_{4}, \mathrm{~T}_{7}\right.$, $\mathrm{T}_{8}, \mathrm{~T}_{9}, \mathrm{~T}_{10}$ ) por meio de regressão.

\section{RESULTADOS E DISCUSSÃO}

A análise de variância (Quadro 1) dos teores totais de metais mobilizados nas colunas e dos valores de $\mathrm{pH}$, obtidos nas soluções percoladas, mostrou diferenças significativas entre os tratamentos, a $1 \%$ de probabilidade. Os valores totais de metais mobilizados nas colunas mostraram uma predominância de $\mathrm{Zn}, \mathrm{Mn}$, $\mathrm{Ni}$ e $\mathrm{Fe}$, sendo que os teores de $\mathrm{Pb}$ foram baixos.

\section{Acidificação das Águas de Drenagem}

$\mathrm{O}$ contraste entre médias dos valores de $\mathrm{pH}(\mathrm{C} 1)$, indica que os tratamentos com camadas de argilas apresentam valores significativamente (1\% probabilidade) menores do que aqueles sem camadas de argila (Figura 2). Quando se considera o contraste que avalia a posição das argilas (C2), observa-se que existe diferença significativa ao nível de $5 \%$ de probabilidade (Quadro 2). Isto indica que a presença das camadas de argilas, contribui para a acidificação das águas de drenagem. Este resultado, embora inesperado, sugere a presença de sulfetos não só nos rejeitos mas também nas argilas.

Para o contraste onde se estuda o efeito de $\mathrm{CaCO}_{3}$ adicionado ao rejeito (C3),observa-se a influência do carbonato na elevação do $\mathrm{pH}$ das águas de drenagem com significância ao nível de $1 \%$ de probabilidade. Da mesma forma, para o contraste onde se estuda o efeito do $\mathrm{CaCO}_{3}$ em presença de camada de argila sobre as cinzas (C4), observa-se que os valores de pH são

Quadro 1: Análise de variância para os teores totais de metais mobilizados e valores de pH nas soluções percoladas das colunas.

Table 1: Analysis of variance for the total amounts of heavy-metals and $\mathrm{pH}$ values in the percolated solutions.

\begin{tabular}{|c|c|c|c|c|c|c|c|}
\hline \multirow[t]{2}{*}{ Fontes de variação } & \multirow[t]{2}{*}{ G. L. } & \multicolumn{6}{|c|}{ Quadrados Médios } \\
\hline & & Zn & Mn & $\mathbf{N i}$ & $\mathrm{Fe}$ & $\mathrm{Pb}$ & $\mathrm{pH}$ \\
\hline Blocos & 2 & $14,15^{\mathrm{ns}}$ & $25,10^{\mathrm{ns}}$ & $9,31^{\mathrm{ns}}$ & $41,79^{\mathrm{ns}}$ & $0,004^{\mathrm{ns}}$ & $0,0105^{\text {ns }}$ \\
\hline Tratam & 10 & $311,34^{* * *}$ & $4304,57^{* * *}$ & $80,19^{* * *}$ & $1085,49^{* * *}$ & $0,0043^{* * *}$ & $16,9719^{* * *}$ \\
\hline Erro & 20 & 4,52 & 61,92 & 6,48 & 114,14 & 0,0003 & 0,0678 \\
\hline $\mathrm{CV}(\%)$ & & 17,53 & 22,66 & 46,98 & 83,71 & 108,27 & 5,54 \\
\hline
\end{tabular}

${ }^{* * \star}$ - significativo a $0,1 \%$ de probabilidade. ns- não significativo até nível de $5 \%$ de probabilidade.

Quadro 2: Análise de variância dos contrastes ortogonais para valores de $\mathrm{pH}, \mathrm{Zn}, \mathrm{Ni}, \mathrm{Fe}$, Mn e Pb nas soluções lixiviadas.

Table 2: Analysis of variance of constrasts for $\mathrm{pH}, \mathrm{Zn}, \mathrm{Ni}, \mathrm{Fe}, \mathrm{Mn}$ and $\mathrm{Pb}$ leached solutions.

\begin{tabular}{cccccccc}
\hline Contraste & G.L. & \multicolumn{5}{c}{ Quadrados Médios } \\
\cline { 3 - 7 } & & $\mathbf{p H}$ & $\mathbf{Z n}$ & $\mathbf{N i}$ & $\mathbf{F e}$ & $\mathbf{M n}$ & $\mathbf{P b}$ \\
\hline C1 & 1 & $20,026^{* *}$ & $1,18^{\mathrm{ns}}$ & $66,59^{* *}$ & $302,01^{\mathrm{ns}}$ & $204,53^{\mathrm{ns}}$ & $0,087^{* *}$ \\
C2 & 1 & $0,217^{*}$ & $0,32^{\mathrm{ns}}$ & $43,72^{* *}$ & $979,30^{* *}$ & $124,24^{\mathrm{ns}}$ & $0,097^{* *}$ \\
C3 & 1 & $22,592^{* *}$ & $1172,31^{* *}$ & $138,38^{* *}$ & $1613,07^{* *}$ & $13366,75^{* *}$ & $0,000^{\mathrm{ns}}$ \\
C4 & 1 & $1,011^{* *}$ & $107,76^{* *}$ & $163,40^{* *}$ & $4132,01^{* *}$ & $2592,84^{* *}$ & $0,0005^{\mathrm{ns}}$ \\
C5 & 1 & $0,070^{\mathrm{ns}}$ & $19,35^{\mathrm{ns}}$ & $1,41^{\mathrm{ns}}$ & $193,03^{\mathrm{ns}}$ & $758,36^{* *}$ & $0,0162^{* *}$ \\
C6 & 1 & $0,017^{\mathrm{ns}}$ & $0,29^{\mathrm{ns}}$ & $1,82^{\mathrm{ns}}$ & $995,44^{* *}$ & $90,07^{\mathrm{ns}}$ & $0,0000^{\mathrm{ns}}$ \\
\hline
\end{tabular}

ns não significativo. * significativo a $5 \%$ de probabilidade. ${ }^{* *}$ significativo a $1 \%$ de probabilidade. 
maiores na presença de $\mathrm{CaCO}_{3}$, com significância ao nível de $1 \%$ de probabilidade. O mesmo já não acontece quando se estuda o efeito do $\mathrm{CaCO}_{3}$ misturado ao rejeito e com camadas de argila sobre e sob as cinzas (C5). Estes resultados indicam que o uso de $\mathrm{CaCO}_{3}$ é eficiente para neutralizar a acidez gerada pela oxidação dos sulfetos presentes no rejeito, conforme admitem váriosos autores (Evangelou 1995; Caruccio \& Geidel 1996). No entanto, a eficiência do $\mathrm{CaCO}_{3}$ em neutralizar a acidez diminui na presença de camadas de argila, conforme se pode observar na Figura 2, comparandose os tratamentos 1 e 4,2 e 5,3 e 6 .

O comportamento do $\mathrm{pH}$ para os tratamentos $1 ; 7$; 4; 8; 9 e 10 que refletem, respectivamente, as doses de $\mathrm{CaCO}_{3}$ equivalente a 0,$0 ; 0,2 ; 0,4 ; 0,6 ; 0,8$ e 1,0 N.C., resulta no modelo quadrático com coeficiente de determinação de $99 \%$.. Esse modelo indica que a dose de $\mathrm{CaCO}_{3}$ equivalente a 0,4 N.C foi suficiente para elevar o pH a 7,0, considerando o tempo de desenvolvimento do experimento. Nestas condições, teoricamente, a solubilização e mobilização dos metais, nas águas de drenagem, fica limitada. Verifica-se, ainda, que os tratamentos 1 e 11, comparados pelo contraste C6 (Figura 2a), apresentam valores de pH que não diferem significativamente. Isto indica que a presença das cinzas não afeta o pH das soluções percoladas; e que as fontes primordiais de acidez encontram-se apenas na argila e rejeito.

\section{Mobilização de Metais nas Colunas de Lixiviação.}

\section{Níquel, Zinco e Ferro}

Observa-se que os teores de $\mathrm{Ni}$ nas soluções percoladas, são influenciados pela presença de camadas

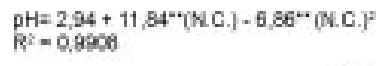

(a)
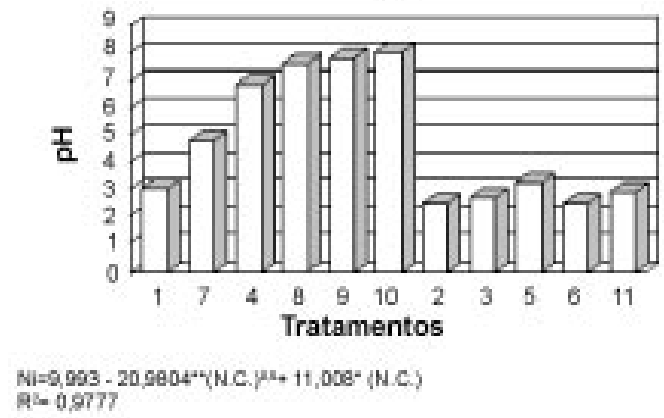

(b)

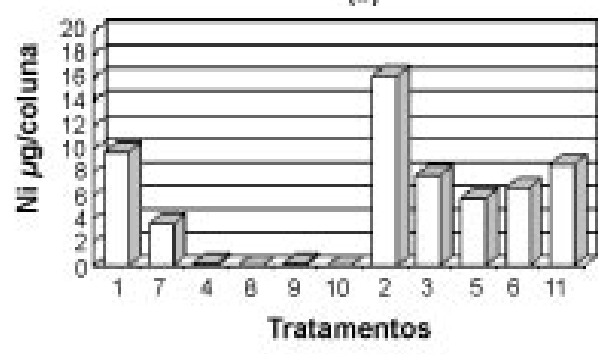

de argila, que contribuem para o aumento dos teores deste metal mobilizado nas colunas (Figura 2b). Isto é evidenciado pelo contraste $\mathrm{C} 1$, cujo efeito é significativo a $1 \%$ de probabilidade (Quadro 2). O efeito da posição das camadas de argila (C2) para estes metais foi significativo a $1 \%$ de probabilidade, com exceção do Zn. Verifica-se que os tratamentos com uma camada de argila sobre as cinzas $\left(\mathrm{T}_{2}\right.$ e $\left.\mathrm{T}_{5}\right)$ apresentaram, em média, maiores teores de $\mathrm{Ni}$ e $\mathrm{Fe}$ na solução percolada do que aqueles com camadas de argila sobre e sob as cinzas $\left(\mathrm{T}_{3}\right.$ e $\mathrm{T}_{6}$ ). Estes resultados (Fig. $2 \mathrm{~b}$ e 2d), aparentemente, são contraditórios, pois se a presença de uma camada de argila contribui para o aumento dos teores de metais, a presença de duas camadas deveria aumentar ainda mais os teores destes nas soluções percoladas. No entanto, verifica-se o contrário. Uma explicação para tal contradição, pode ser devido aos menores volumes de solução coletados das colunas que receberam duas camadas de argila (Quadro 3).

No contraste que estuda o efeito do $\mathrm{CaCO}_{3}$ misturado ao rejeito (C3), observa-se nitidamente a atuação do carbonato limitando a mobilização dos metais. Tal efeito não se restringiu apenas a $\mathrm{Zn}, \mathrm{Ni}$, e $\mathrm{Fe}$, mas a todos os metais estudados.

No estudo do efeito de $\mathrm{CaCO}_{3}$ dentro de tratamentos com camada de argila sobre as cinzas $\left(\mathrm{T}_{5}\right.$ versus $\left.\mathrm{T}_{2}\right)$, representado pelo contraste $\mathrm{C} 4$, observou-se que o tratamento com $\mathrm{CaCO}_{3}$ foi mais eficiente em diminuir os teores de metais mobilizados na solução, com significância ao nível de $1 \%$ de probabilidade. O mesmo efeito não foi observado quando se estudou a presença de $\mathrm{CaCO}_{3}$ misturado ao rejeito, com camadas de argila sobre e sob as cinzas (C5) cujos contrastes não foram

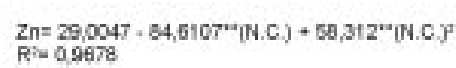

(c)

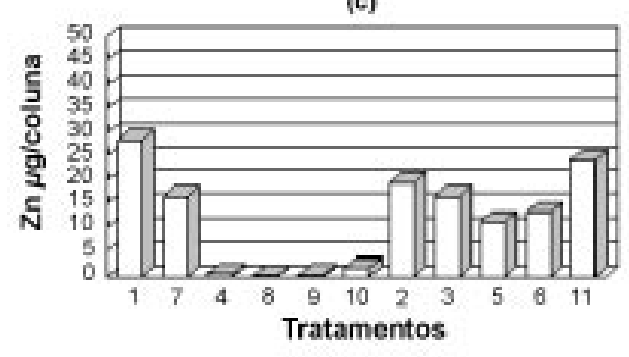

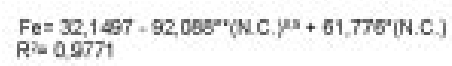

(d)

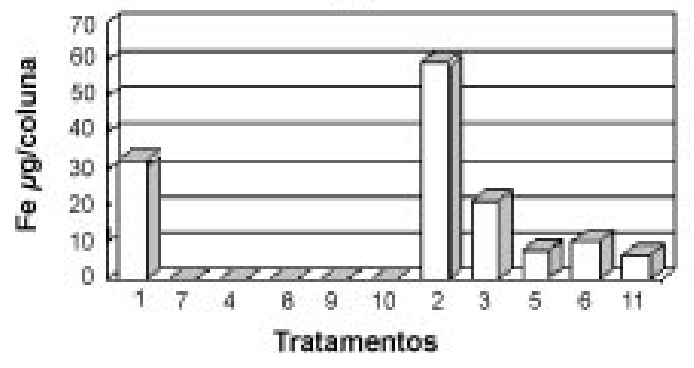

Figura 2: Valores de pH, Ni, Zn e Fe nas soluções percoladas das colunas para os diferentes tratamentos.

Figure 2: $\mathrm{pH}, \mathrm{Ni}, \mathrm{Zn}$ and $\mathrm{Fe}$ values in the percolated solution for all treatments. 
Quadro 3: Volumes de solução coletada, até o quarto dia após a simulação das chuvas nas colunas de lixiviação para os diferentes tratamentos.

Table 3: Volums of colected solution, up to the fourth day after simulated rainfall, for all treatments.

\begin{tabular}{cccccccccc}
\hline Trat & C S 1 & C S 2 & C S 3 & C S 4 & C S 5 & C S6 & C S 7 & C S 8 & Total \\
\cline { 2 - 9 } & & & \multicolumn{7}{c}{ ml } \\
\hline $\mathrm{T}_{1}$ & 593 & 543 & 526 & 526 & 496 & 740 & 740 & 740 & 4904 \\
$\mathrm{~T}_{2}$ & 650 & 170 & 450 & 450 & 183 & 740 & 500 & 450 & 3593 \\
$\mathrm{~T}_{3}$ & 150 & 80 & 175 & 150 & 57 & 200 & 60 & 150 & 1022 \\
$\mathrm{~T}_{4}$ & 710 & 740 & 518 & 510 & 546 & 593 & 463 & 463 & 4573 \\
$\mathrm{~T}_{5}$ & 406 & 283 & 666 & 666 & 396 & 740 & 466 & 466 & 4089 \\
$\mathrm{~T}_{6}$ & 340 & 313 & 250 & 246 & 406 & 363 & 243 & 740 & 2901 \\
$\mathrm{~T}_{7}$ & 593 & 626 & 740 & 740 & 626 & 740 & 500 & 740 & 5305 \\
$\mathrm{~T}_{8}$ & 740 & 740 & 606 & 570 & 740 & 740 & 383 & 400 & 4919 \\
$\mathrm{~T}_{9}$ & 740 & 740 & 740 & 740 & 346 & 740 & 366 & 740 & 5152 \\
$\mathrm{~T}_{10}$ & 740 & 740 & 740 & 740 & 740 & 740 & 466 & 740 & 5646 \\
$\mathrm{~T}_{11}$ & 496 & 380 & 296 & 305 & 333 & 150 & 210 & 233 & 2403 \\
\hline
\end{tabular}

C S - Chuva simulada

significativos até o nível de $5 \%$ de probabilidade. Estes resultados novamente demonstram que a eficiência do $\mathrm{CaCO}_{3}$ diminuiu na presença de camadas de argila, conforme já discutido para a variável $\mathrm{pH}$.

A comparação entre médias dos tratamentos com presença e com ausência de cinzas $\left(T_{1}\right.$ e $T_{11}$, respectivamente) mostra que os metais $\mathrm{Zn}$, Ni e Fe apresentam tendências diferenciadas. Verificou-se um aumento nos teores de $\mathrm{Fe}$ onde a cinza se encontra presente, com significância ao nível de $1 \%$ de probabilidade. Já o $\mathrm{Zn}$ e o $\mathrm{Ni}$ não apresentaram variações significativas até o nível de 5\%. Estes resultados indicam que não são as cinzas de combustão do carvão a principal fonte de Zn e Ni e sugerem que a simples retirada das cinzas não é suficiente para conter a contaminação das águas de drenagem da mina.

$\mathrm{O}$ reflexo do comportamento dos valores destes metais para os tratamentos $1,7,4,6,8,9$ e 10 que indicam, respectivamente as doses de $\mathrm{CaCO}_{3}$ equivalentes a 0,$0 ; 0,2 ; 0,4 ; 0,6 ; 0,8$ e 1,0 N.C., resultam nos modelos: quadrático para o $\mathrm{Zn}$, com coeficiente de determinação próximo a $97 \%$ e raiz quadrada para $\mathrm{Ni}$ e Fe com coeficiente de determinação próximo a $98 \%$. Em todos os casos, a exemplo do que se verificou para a variável $\mathrm{pH}$, a dose de $\mathrm{CaCO}_{3}$ correspondente a 0,4 N.C. foi suficiente para reduzir a níveis próximos de zero os teores de $\mathrm{Zn}$; Ni e Fe nas soluções percoladas.

\section{Manganês}

Quando se estuda o efeito da presença e posição de camadas de argila (C1 e C2), observa-se que os teores de $\mathrm{Mn}$, ao contrário do que se verificou para $\mathrm{Ni}$ e $\mathrm{Fe}$, não se alteram significativamente, até o nível de $5 \%$ de probabilidade (Quadro 2).

No estudo da presença de $\mathrm{CaCO}_{3}$ misturado ao rejeito sem camadas de argila (C3), é evidente a diminuição do teor deste metal na solução quando o carbonato foi adicionado (Figura 3), ao nível de significância de $1 \%$ de probabilidade. O mesmo se verificou na presença de camadas de argila sobre as cinzas (C4) e em presença de argila sobre e sob as cinzas (C5).

O contraste que avalia o efeito da presença de cinzas (C6) mostra que os teores deste elemento não diferem significativamente, ao nível $5 \%$ de probabilidade, entre os tratamentos $\mathrm{T}_{1}$ e $\mathrm{T}_{11}$, da mesma forma que ocorreu para o $\mathrm{Zn}$ e o $\mathrm{Ni}$.

O comportamento deste elemento para os tratamentos 1, 7, 4, 8, 9 e 10 é descrito pelo modelo raiz quadrada com coeficiente de determinação de 99 $\%$.

\section{Chumbo}

O efeito da presença de camadas de argilas é evidenciado pelo contraste $(\mathrm{C} 1)$, onde os tratamentos com camadas de argilas superaram em teor médio de $\mathrm{Pb}$ os valores apresentados pelos tratamentos sem camadas de argila, ao nível de significância de $1 \%$ de probabilidade.

Ao se comparar o efeito posição das camadas de 
$M n=94,54-236,294^{* *}(\mathrm{~N} . \mathrm{C} .)^{25}+145,137^{* *}(\mathrm{~N}$ C. $)$ $R^{2}=0,9927$

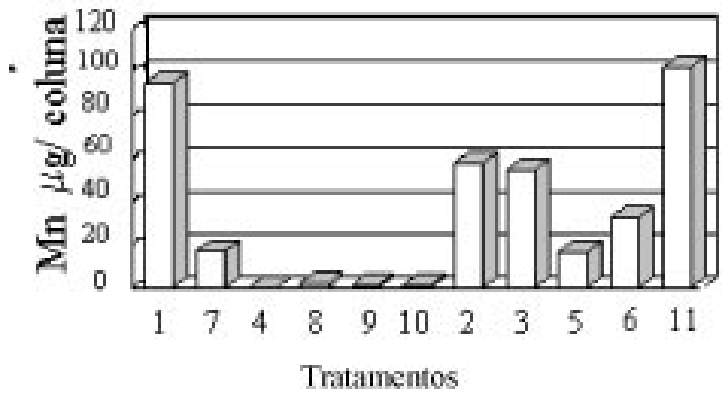

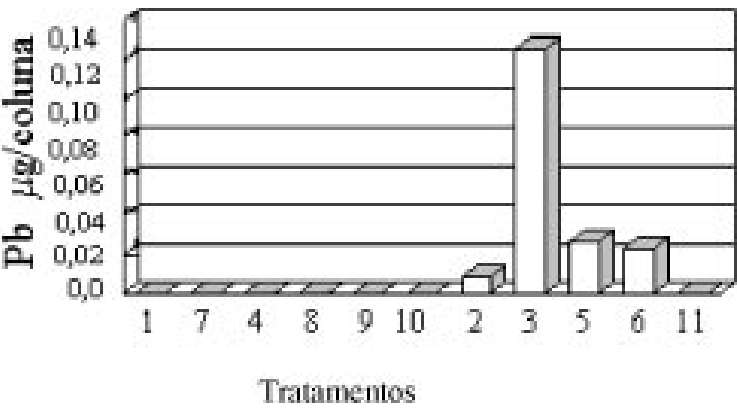

Tratamentos

Figura 3: Valores totais de Mn e Pb nas soluções percoladas das colunas para os diferentes tratamentos. Figure 3: Total amounts of $\mathrm{Mn}$ and $\mathrm{Pb}$ in ther percolated solution in the treatments.

argila (C2), observa-se que aqueles tratamentos em que as camadas de argila localizaram-se sobre e sob as cinzas, superaram em teores médios de $\mathrm{Pb}$ na solução, com significância ao nível de $1 \%$ de probabilidade.

Quando a comparação é feita entre tratamentos com $\mathrm{CaCO}_{3}$ misturados ao rejeito e tratamentos sem $\mathrm{CaCO}_{3}$ (C3), não houve diferença significativa até o nível de $5 \%$ de probabilidade, em razão da virtual ausência de $\mathrm{Pb}$ nas soluções percoladas para todos os tratamentos sem camadas de argila. Estes resultados evidenciam a influência das camadas de argila contribuindo para valores mais elevados deste metal (Figura 3). Este fato pode ser explicado pela adsorção preferencial deste elemento na argila presente nestes depósitos, a qual constitui-se predominantemente de caulinita. Vale lembrar que a seletividade por chumbo, nos processos de adsorção em caulinitas, é um fato bem estabelecido, conforme admite ALLOWAY (1990).

$\mathrm{O}$ efeito de $\mathrm{CaCO}_{3}$ dentro dos tratamentos com camadas de argila apenas sobre as cinzas (C4) mostra que o elemento $\mathrm{Pb}$ apresentou valores médios mais altos, para os tratamentos com $\mathrm{CaCO}_{3}$, sendo que esta diferença não é significativa até o nível de 5\% de probabilidade. Para o efeito de $\mathrm{CaCO}_{3}$ dentro de tratamentos com camadas de argila sobre e sob as cinzas (C5), observa-se que o $\mathrm{Pb}$ apresentou valores médios superiores no tratamento onde não houve adição de $\mathrm{CaCO}_{3}$ misturado ao rejeito, com diferença significativa ao nível de $1 \%$ de probabilidade.

Quanto ao efeito da presença de cinzas (C6) observa-se que não houve diferença significativa até o nível de $5 \%$ de probabilidade.

\section{CONCLUSÕES}

Os resultados obtidos permitiram concluir que:

a) Existe mobilização de metais pesados com potencial para contaminação das águas de drenagem na mina.

b) O uso de camadas de argila não foi eficiente em minimizar a acidificação e a movimentação de metais pesados nas soluções percoladas nas colunas. c) As colunas tratadas apenas com carbonato de cálcio em quantidade igual ou maior do que 0,4 vezes a necessidade de calcário, não mostraram movimentação evidente de metais pesados na solução percolada.

d) As colunas onde foram utilizadas juntamente as argilas e $\mathrm{CaCO}_{3}$ mostraram uma mobilização acentuada de metais, indicando que a presença de camadas de argila diminui a eficiência dos carbonatos em neutralizar a acidez.

e) As cinzas da combustão do carvão não constituem a única fonte de contaminação com metais pesados, sendo que os estéreis apresentaram teores elevados de $\mathrm{Mn}, \mathrm{Zn}$, Ni e $\mathrm{Fe}$, e que as argilas constituíram a principal fonte de $\mathrm{Pb}$.

\section{REFERÊNCIAS BIBLIOGRÁFICAS}

ABOARRAGE, A .M. \& LOPES, R. C. - 1986- Projeto a Borda Leste da Bacia do Paraná: Integração Geológica e Avaliação Econômica: Relatório Final. Porto Alegre, DNPM/CPRM. v1 $15 \mathrm{p}$.

ALLOWAY, B. J. Soil processes and the behaviour of metals. In: Alloway, B. J. Ed. Heavy metals in soils. John wiley, New York, 1990. P 7-28.

BRASIL, 1987 Departamento Nacional de Produção Mineral. Perfil Analítico do Carvão . D.N.P.M. Porto Alegre, (Boletim 6.). 48 p.

CARUCCIO, F. T. \& GEIDEL, G. Acid mine drainage; the laboratory \& field settings. Workshop $13^{\text {th }}$ Annual national meeting of the American Society for surface mining and reclamation.. Knoxville, Tenesse, USA, 1996. 58p.

EMPRESA BRASILEIRA DE PESQUISA AGROPECUARIA 1979 - Serviço Nacional de Levantamento e Conservação de Solos EMBRAPA - Manual de métodos de análises de solo. Rio de janeiro, S.N.L.C.S., n.p.

EVANGELOU, V. P. Pyrite oxidation and it's control. CRC press. New York, 1995. 293p.

FLORES, E. M. 1990. Utilização de amostras ambientais das regiões de Candiota e Charqueadas (RS) como bioindicadores de poluição. Universidade Federal de Santa Maria RS, (tese M.S.).

SINGER, P. E. \& STUMM, W. Acid mine drainage: The rate determining step. Science, 167 1121-1123, 1970.

ZANELLA, R. 1988. Investigação dos problemas ambientais relacionados com a exploração intensiva e queima de carvão termoelétrico em Candiota RS. Universidade Federal de Santa Maria RS, (tese M.S). 